\title{
Modeling Techniques Suitable for CAD Based Design of Internal Matching Networks of High-Power RF/Microwave Transistors
}

\author{
Peter H. Aaen, Member, IEEE, Jaime A. Plá, Member, IEEE, and Constantine A. Balanis, Life Fellow, IEEE
}

\begin{abstract}
A scalable and accurate simulation technique to be used for the computer-aided design of matching networks employed within high-power RF transistors is presented. A novel measurement methodology is developed and utilized during the validation of the proposed analysis approach. Appropriate segmentation techniques were developed, which are consistent with the design approach of the high power transistor, that take into account the overall complexity of the internal match of most modern RF high power transistors, while preserving important electromagnetic interactions. By being able to properly decouple the linear portion of the overall packaged transistor model, an objective accuracy assessment via the comparison of measured vs. simulated results of the internal matching network was accomplished. The level of accuracy obtained provides credence to the idea of a full CAD driven design process of the internal match of high-power RF transistors.
\end{abstract}

Index Terms-High-power RF transistors, CAD techniques, bonding-wires, MOS capacitors

\section{INTRODUCTION}

A $\mathrm{S}$ the complexity of wireless infrastructure systems continue to evolve, more companies are relying on computeraided design (CAD) methodologies to dramatically reduce time-to-market and to increase design robustness. For semiconductor vendors, the ability to provide accurate non-linear electro-thermal models in a timely fashion has become an important differentiator between competing suppliers.

At the heart of the wireless infrastructure power amplifier is the high-power RF transistor. The silicon LDMOS transistor is the technology of choice, due to its superior electrical and thermal performance and the inherent economic advantages that silicon wafer manufacturing offer due to its economy of scale over alternative compound semiconductor technologies. To satisfy the high power levels required in todays power amplifiers, Laterally Diffused Metal Oxide Semiconductor (LDMOS) transistors need to be of high gate periphery. In addition, to facilitate their ease of use, the LDMOS die impedances are increased by means of complex and very lowloss matching networks inside high performance ceramic or plastic packages [1].

The development of non-linear electro-thermal models for these packaged transistors taxes the most sophisticated measurement and simulation techniques [1]-[7]. After a model has been completed, the final process of comparing the model against an independent set of measurements (i.e. validating the model) begins. During this process, the modeling engineer is challenged with deciphering the various sources of inaccuracies. In the most fundamental form, the sources of inaccuracies can be categorized as originating from the electrical or thermal sections of the packaged transistor model; furthermore a subsequent subdivision can be made between the linear and non-linear sections of the electrical and thermal portions of the model. It is extremely advantageous to decouple the thermal and electrical effects as well as the linear and non-linear problems, so modeling resources can be strategically focused and the model improved.

Without the ability to decouple the error sources, an often employed approach is to optimize the equivalent circuit parameters (ECPs) of the model until its performance matches measured data. When a significant number of ECPs (i.e. free parameters or degrees of freedom of the model) are simultaneously optimized, the circuit essentially memorizes the data to which it has been compared. The resulting model is an adhoc fit which is only valid over the range of measurement data to which it is optimized and its predictive capability over the non-measured data space is often seriously compromised. With the increasing proliferation of modulation schemes and circuit design topologies (e.g. Doherty, Digital Pre-Distortion, Feed-Forward, etc.) it is extremely difficult to have a model generated in this manner which describes all of these operating conditions successfully.

The need for an alternative approach in which, the linear and non-linear portions of the circuit are separated and examined is essential, at which point the accuracy of the specific linear or non-linear modeling techniques and/or procedures can be examined. The focus of this paper is to perform an in-depth comparison between the measured and simulated electrical linear portions of the circuit, specifically, the linear section of the packaged transistor model.

Full-wave simulations of the complete matching network were thought to be prohibitively expensive and approximations frequently were employed. However, through the development of segmentation procedures, it is possible to divide the entire packaged transistor into portions and then appropriately recombine the modeled parts [8].

In this paper, we present for the first time the development of a full-wave methodology to be used for the computer-aided design of matching networks employed within high-power RF transistors. Section II discusses the measurement methodology utilized during the validation and development of simulation techniques of the high-power RF/Microwave transistors. The overall segmentation of the matching network constituent components and the developed simulation methodology are covered in Section III. A comparison of the measured vs. 
simulated results is examined and presented in Section IV, while the work concludes in Section V.

\section{Measurement Methodology}

The design of test-fixtures for the measurement of the S-parameters of packages used in the construction of RF power amplifiers is a challenging task. The requirements of a repeatable, high-frequency capable and wide-bandwidth testfixture are at odds with a package that is designed for highvolume manufacturing. Nevertheless, the development of such a test-fixture is a necessity for the development of CADbased design techniques. In order to better understand these difficulties, it is instructive to examine the construction of an RF power package.

A photograph of an RF transistor with internal matching is provided in Fig. 1. In general, the width of the package leads approximately match the combined width of the dies mounted within the package. Thus, for high-power transistors, which typically contain three active dies, the leads can be as wide as 500 mil. For these packages the ceramic window-frame is typically 20 mil thick and the flange is approximately $60 \mathrm{mil}$ thick.

When attempting to obtain S-parameters of a device using one of these packages, it is often difficult to obtain accurate measurements. The difficulty arises primarily for three reasons. The first is that the packages are designed to be used in lowcost high-volume manufacturing lines in which the package will be soldered onto a circuit board. Since we desire a testfixture which can be reused, soldering each packaged device into the test-fixture is not feasible. As such, there is a variance in the package dimensions due to manufacturing tolerances which allows the package to change position within the testfixture. The second difficulty is that the transmission lines used in the test-fixture, for S-parameter measurement, are designed such that the microstrip discontinuity between the package and the fixture is minimized by making the transmission line width the same as the package lead width. These wide lines complicate the test-fixture design since a $50 \Omega$ characteristic impedance, on a given substrate, can only be maintained by adjusting the thickness of the substrate [5]. The third difficulty arises from the thickness of the package flange. In many instances, thickness of the flange requires a recessed area in the heat-sink in order for the leads of the package to be able to connect to the test-fixture. This abrupt change in current flow creates an electrical discontinuity which affects the circuit performance [9]. Furthermore, the wide transmission lines reduce the maximum usable frequency of the test-fixture. The design of a test-fixture that overcomes these measurement issues is presented in the following sub-section.

\section{A. GSG Probable Test-Fixture}

The design of the test-fixture employs ground-signal-ground (GSG) probes and standard thickness substrates. The testfixture is constructed of a single printed circuit board (PCB) soldered to a metal carrier, which has a recessed area to accommodate the package flange as indicated in Fig. 2(a). The depth of the recessed area is such that the leads of the

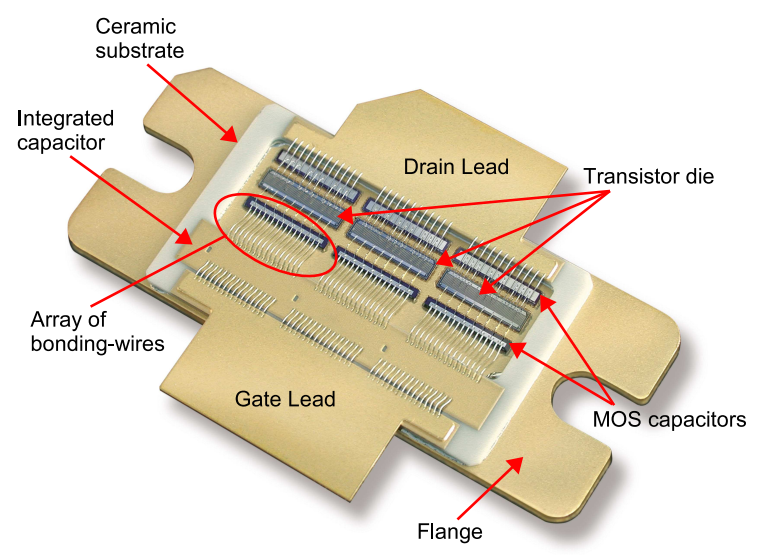

Fig. 1. The complexity of the internal construction of an $2.1 \mathrm{GHz} 140 \mathrm{~W}$ LDMOS transistor is shown in this photograph. The width of the package lead is approximately $500 \mathrm{mil}$.

package rest on top of the test-fixture when the package flange is securely fastened to the metal-carrier. An exploded diagram, indicating how the package is mounted within the fixture, is provided in Fig. 2(b). The device-under-test is secured within the fixture by a specially machined plastic block. The block is bolted on top of the package and is designed to push down on the package so that a conductive connection is established between the package flange and the metal carrier. The block also forces contact between the package leads and the microstrip transmission lines of the test-fixture. Both the plastic block and the package leads are included in the simulation methodology to ensure consistency between measurement and simulation.

The issues with fixture assembly and repeatability are avoided by manufacturing the calibration standards on a single PCB. Thus, no repeated assembly of the fixture is required as is the case with a coaxial connector based $50 \Omega$ test-fixture. The interconnection between the VNA and the test-fixture is accomplished through the use of GSG probes. These probes are attached to micro-manipulators as part of a probe-station and are used to form very repeatable connections.

A substrate thickness of 20 mil was selected since it minimizes the discontinuity in the ground-plane because it is the same as the ceramic window-frame of the package. However, a consequence of using the thinner substrates and requiring that the widths of the transmission line and package leads match is that the characteristic impedance of the line is significantly lower than $50 \Omega$.

Unfortunately, it is difficult to obtain S-parameters of a device which is embedded in a non-50 $\Omega$ test-fixture since a large reflection coefficient is introduced by the mismatch in characteristic impedances between the microstrip transmission lines and the coaxial cables [6]. The difficulty arises when de-embedding the test-fixture and attempting to distinguish the reflections introduced by the test-fixture as opposed to those from the device. A test-fixture which introduces large reflections degrades the dynamic range of the measurement and has the potential to introduce large errors. 
To reduce the mismatch between the $50 \Omega$ coaxial environment and the low-impedance transmission line, distributed planar matching networks were used. The Tschebyscheff transformer was selected for this application since it provides the largest bandwidth for a given number of impedance transformer sections, and it is easily manufactured. Its selection assists in keeping the size of the test-fixture as small as possible. The last length of the Tschebyscheff transformer is connected to a section of transmission line selected to match the lead width of the packaged transistor, as shown in Fig. 2(a). Thus, the transformation ratio is dictated by the width of the package lead and the substrate characteristics selected.

A full set of standards, to perform a TRL calibration, was manufactured and photographs of the three standards used during the calibration procedure are shown in Figures 3(a) and 3(b). Note that the thru standard consists of the two sides of the test-fixture placed back-to-back, while the line standard consists of two transformers separated by a length of transmission line. The reflect standards are transformers which have either open or short circuits at the ends of the fixture.

The calibration procedure for this test-fixture is similar to one employed for load-pull measurements [6]. The outer shell of the calibration is based on a $50 \Omega$ characteristic impedance while the inner shell is based on the characteristic impedance of the lines used during the TRL calibration. The inner shell calibration allows the determination of the S-parameters of each half of the fixture.

\section{Segmentation and Simulation Methodology}

The matching network of the packaged transistor is a complex electromagnetic environment that contains hundreds of bond-wires and multiple MOS capacitors all in close proximity. The behavior of the matching network is controlled by the three dimensional shape of the bonding-wires and the values of the capacitors. The thickness of the thin layer of oxide (e.g. 0.1-0.2 $\mu \mathrm{m}$ ) and the metal plate area determine the capacitance of the MOS capacitor, while the thickness of the package leadframe and the lead-width determine the capacitance of the package. The critical dimensions controlling these capacitors range from approximately $1 \mathrm{~cm}$ to $0.1 \mu \mathrm{m}$. For structures with these large differences in critical dimensions (i.e. dimensions which affect circuit performance) and the requirement of incorporating the three-dimensional geometry of the bondingwire, it is very difficult to simulate the entire matching network at once using a single simulator and/or a single simulation run. Devising an appropriate segmentation strategy is of paramount importance to be able to properly trade-off simulation time and accuracy. In general, if simulation time is not an issue, simulations are often performed by incorporating as much as possible of the structure. This allows the analysis to include all possible electromagnetic interactions. Conversely, if the computational resources are limited or the overall complexity of the problem is such that it requires to be analyzed in pieces, a systematic and rigorous segmentation scheme must be utilized.

There are two major issues that exist when attempting to implement a segmentation approach to simulate a large

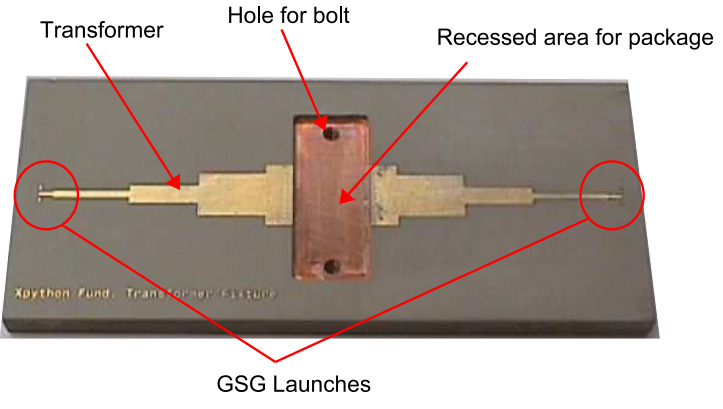

(a) Empty test-fixture

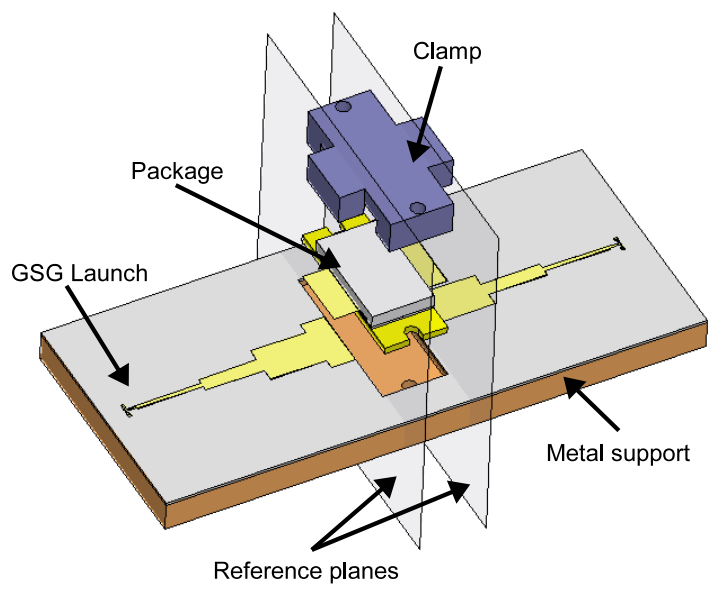

(b) A packaged device within the test-fixture

Fig. 2. A Photographs test-fixture employing Tschebyscheff impedance transformers and an exploded illustration showing how the packaged transistor is held in the fixture by a plastic clamp.

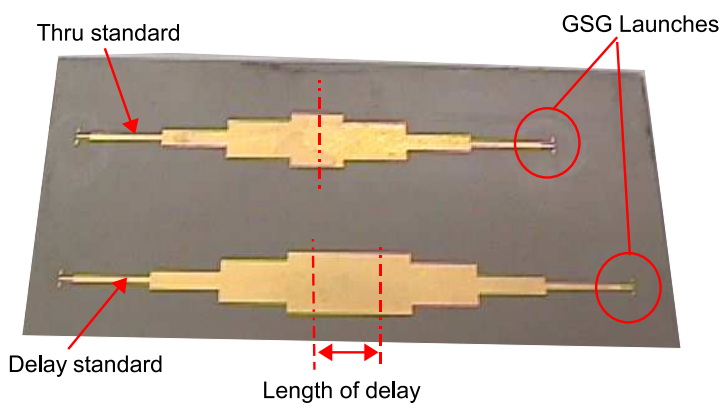

(a) Transmission line standards

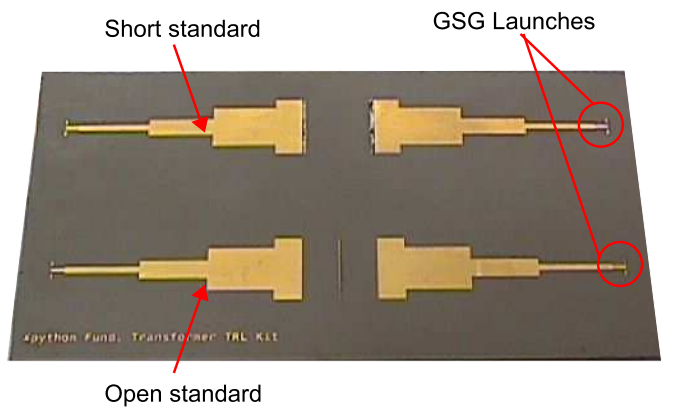

(b) Reflect standards

Fig. 3. Photographs of the TRL standards used during the calibration of the test-fixture. 


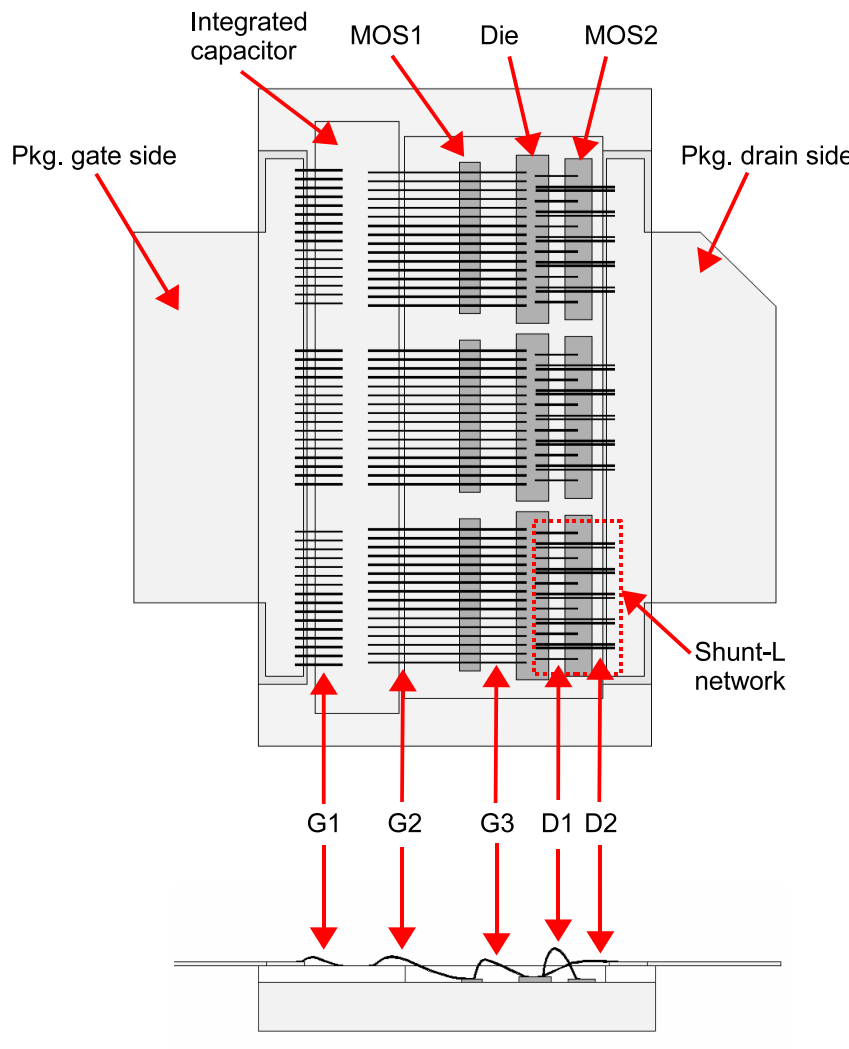

Fig. 4. Top-down view of the test-package.

complex circuit. The first is to devise a set of procedures which allows the individual circuit components to be separated from one another. The separation procedure must not perturb the behavior of the components; i.e., the component when analyzed by itself must operate the same as it does within the larger device. The critical issue with the segmentation approach is that the planes at which the circuit is divided must be carefully selected such that the field configurations on either side of the plane match. Otherwise, a discontinuity is artificially added to the analysis results. The second issue is that any interelement coupling, such as mutual inductance between arrays of bonding-wires, must be properly characterized [1].

For the three-dimensional portions of the circuit (i.e. bondwires), the high-frequency structure simulator $\left(H F S S^{\mathrm{TM}}\right)$ from Ansoft Inc., Pittsburgh, PA, is employed. This simulator is a commercially available implementation of the finite-element method. For the planar portions of the circuit (i.e. MOS capacitors and package), the method-of-moments $\mathrm{em}^{\mathrm{TM}}$ simulator, available from Sonnet Software Inc., North Syracuse, NY, is used. The segmentation procedures used through-out this paper were previously developed and demonstrated by the authors [1].

\section{RESUlts}

All of the analysis and measurement techniques investigated thus far have been developed with the objective of generating a model of the package and matching networks of a high-power RF power transistor. To demonstrate the applicability of these techniques, we generated a model of the matching network for a transistor currently being manufactured. The selected transistor is designed for use within W-CDMA base stations operating in a frequency range from $2.11-2.17 \mathrm{GHz}$. The high-power silicon LDMOS transistor is capable of 30 Watts average output power, $15 \mathrm{~dB}$ power gain, $30 \%$ drain efficiency and $-37 \mathrm{dBc}$ intermodulation distortion product when tested using a two carrier W-CDMA excitation. Under the same tuning conditions, the continuous-wave $(\mathrm{CW})$ output power capability of the transistor exceeds 140 Watts at its $1-\mathrm{dB}$ gain compression point.

The intricate matching circuitry formed by the bondingwires and MOS capacitors is illustrated in Figs. 1 and 4. Rows of parallel bonding-wires form arrays which interconnect the die, MOS capacitors and the package. The packaged-transistor contains three active dies each having a gate periphery of approximately $80 \mathrm{~mm}$. The matching network is composed of the package, 6 MOS capacitors, a capacitor integrated inside the window-frame and 189 bonding-wires. All bonding-wires have a diameter of $50 \mu \mathrm{m}$ and are made of aluminum. The matching networks for this transistor are designed by specifying the values of the MOS capacitors and by specifying the number of bonding-wires, controlling the three-dimensional shape of the bonding-wire and the distance between neighboring wires.

Two types of MOS capacitors are employed in this device; a $22 \mathrm{pF}$ capacitor is used to create part of the matching network on the gate-side of the transistor and a $350 \mathrm{pF}$ capacitor is used on the drain-side. The $350 \mathrm{pF}$ capacitor forms a part of the matching network termed shunt-L, as indicated in Fig. 4. This part of the network is designed such that the array of bonding-wires attached to the drain resonates out the drain-source capacitance $\left(C_{d s}\right)$. The value of the MOS capacitor on the drain-side must be large enough so that within the operating bandwidth the shunt wires are effectively shorted to ground, while still blocking the flow of DC current. To compare simulated and measured results, a special version of the transistor was manufactured which contained no active devices. The silicon LDMOS transistors were replaced with conductive metal blocks approximately the same size as the die. Replacing the die with highlyconductive metal blocks converts the package and matching networks into a high quality-factor $(\mathrm{Q})$ resonator which is very sensitive to the performance of the individual circuit elements [10]. Measurement of a resonator of this type is necessary due to the inherent low loss of the MOS capacitor and bond wire elements. In this manner, the vector-network analyzer can be used to measure directly the S-parameters of the passive structure. Alternatively, one could measure the bias dependent S-parameters of the entire transistor. However, in order to compare the measured and modeled S-parameters, a bias dependent linear model of the silicon LDMOS transistor would have to be combined with the model of the package and matching network. By removing the need to use the bias dependent linear transistor model, we have effectively decoupled the problem of assigning error between the passive constituents of the packaged transistor (air cavity ceramic package, bond wires and MOS capacitors) and the active components (silicon LDMOS die).

The task of generating a model for this device begins 


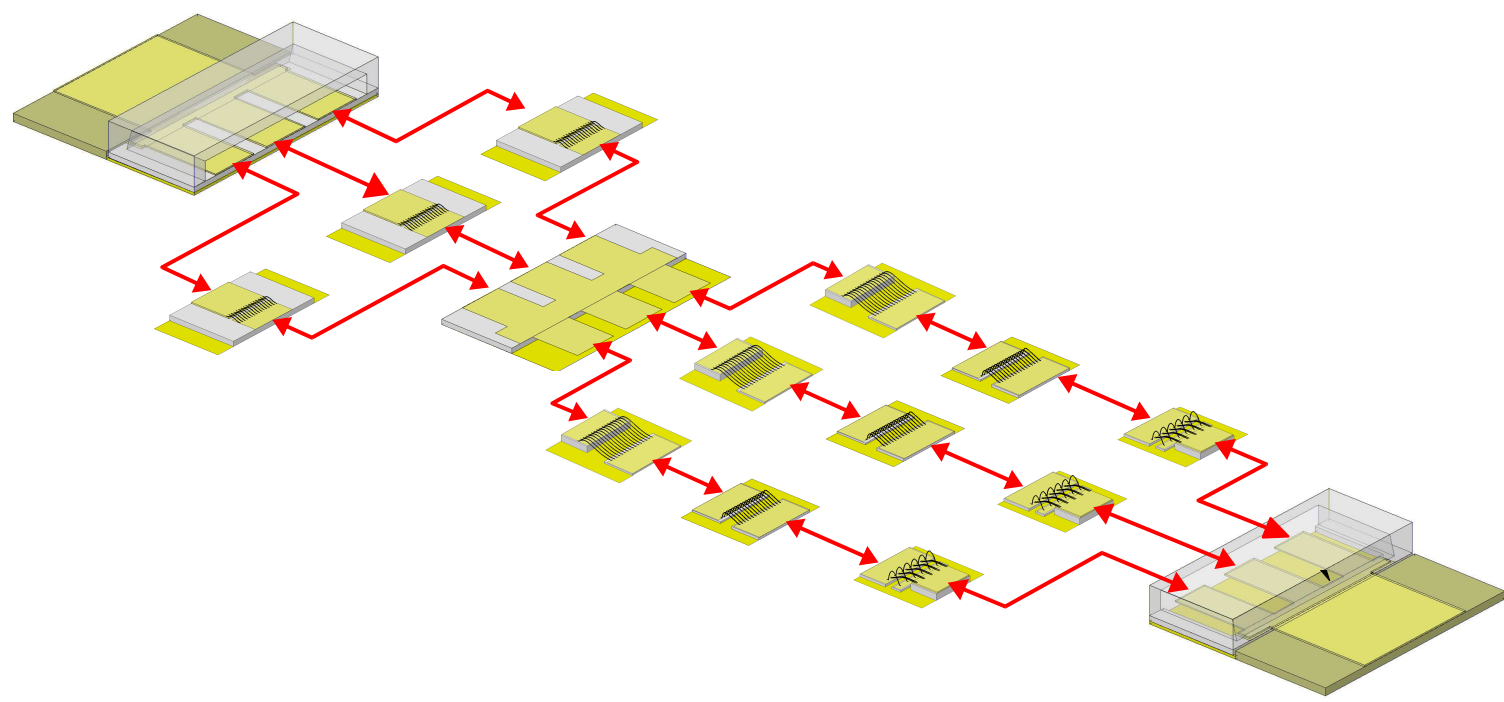

Fig. 5. An illustration of the individual components of the test-package and how the segments fit together to form the model of the entire test-package.

with capturing the geometry of the bonding-wires and the relative positions of the MOS capacitors and conductive blocks within the package. A single wire in each array was selected to represent all other wires. While slight variations due to manufacturing tolerances in the geometrical profiles exist, they were not accounted for and are considered a source of small error in the overall problem accuracy. In total 12 simulations were required to characterize the total device. An illustration outlining the various simulations and how they represent the entire packaged-transistor is provided in Fig. 5. Once all of the simulations were completed, the results were incorporated into a linear circuit simulator. A schematic representing the final model is provided in Fig. 6.

Measurements of the device were performed and a comparison between measured and simulated S-parameters and computed conservation factor is presented in Figs. 7-12. Recall that the features shown in these figures are of the internal matching network without the active semiconductor device inside the air cavity ceramic package. Several important electrical characteristics are observed which highlight the predictive capability of the passive component models. For example, the high Quality $(\mathrm{Q})$ Factor nature of the constitutive components of the matching network, i.e., the array of bond wires, MOS capacitors and air cavity package, is demonstrated by the sharpness of the resonances in the magnitude of $S_{11}$ as shown in Fig. 7. An accurate determination of the inductance of the wire bond arrays (including mutual inductance effects), the capacitance of the MOS capacitors and the effects of the package are necessary to be able to match the exact resonance frequency, while an accurate prediction of the loss determines the degree of agreement of the depth of the resonances. The ability to predict the overall loss of the network is also observed by plotting the conservation factor of the network (Fig. 12). Another important aspect of the electrical performance of the high-power discrete transistor's matching network is the level of isolation between the input and the output sections of the matching network. The overall isolation of the packaged transistor is governed by contributions from the active semiconductor device and the input and output matching networks surrounding it. The active device contribution to the isolation between the input and the output is primarily due to the LDMOS gate-to-drain capacitance and any inductance and resistance connected to the intrinsic source terminal of the transistor. The matching network contribution to the packaged device isolation is mostly due to the inductive coupling between the inner most bond-wire arrays connected to the gate and drain transistor manifolds; it is this level of isolation which can be observed in Fig. 9. Notice that resonances are observed at the same frequencies in which the input matching network is resonant as shown in Fig. 7 as well as a lower frequency resonance due to the interaction of the output's series and shunt inductance elements. The low frequency resonance can also be observed in the magnitude of the output reflection coefficient as shown in Fig. 10. The developed modeling and simulation methodologies previously outlined provide a high-level of accuracy on predicting the isolation contributions from the low-loss high-Q matching networks inside the air-cavity ceramic package. Finally, good agreement is shown between the measured and simulated phase of the input and output return loss of circuit as shown in Fig. 8 and Fig. 11.

As shown, excellent agreement is seen between all measured and simulated results, demonstrating that the matching networks found within high-power RF/microwave transistors can be accurately simulated using full-wave simulators. These simulation techniques effectively reduce the reliance on design methodologies employing significant iterations via manufacturing, thus enabling CAD based design.

\section{CONCLUSIONS}

We have presented a full-wave methodology to be used for the computer-aided design of matching networks employed within high-power RF transistors. The measurement methodology utilized during the validation and development 


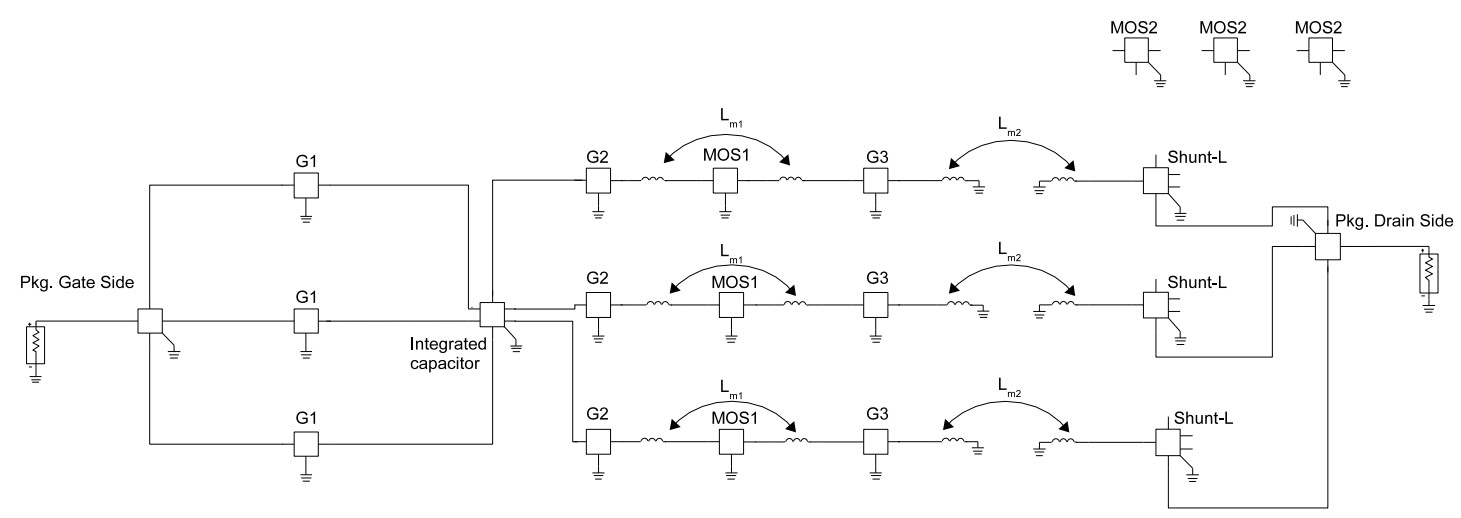

Fig. 6. The final equivalent network. Each box represents the S-parameters of a section of the matching network. Although no direct connection is indicated in the simplified diagram, the boxes labeled as MOS2 are connected to the Shunt-L bond-wires.

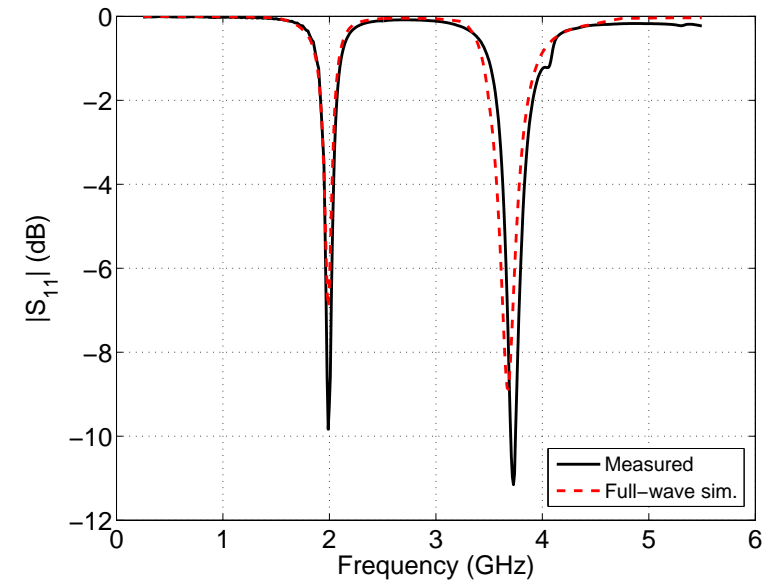

Fig. 7. The magnitude of the simulated and measured input reflection coefficient of the device.

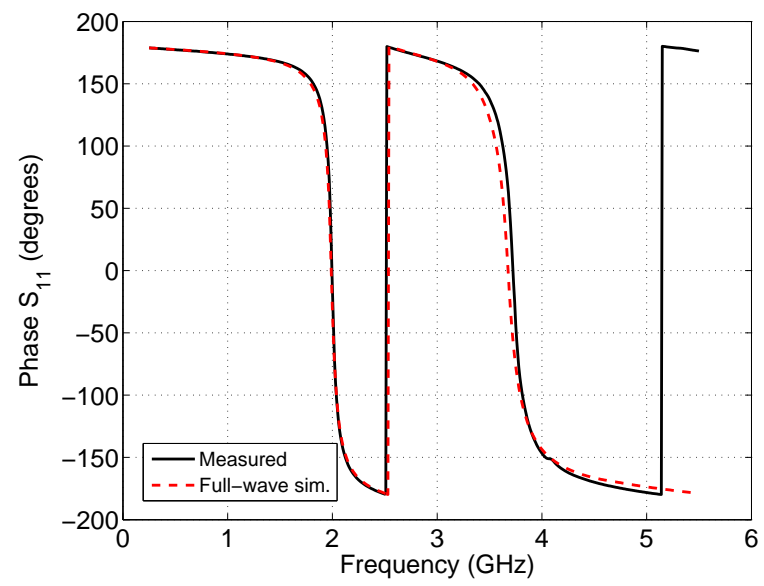

Fig. 8. The phase of the simulated and measured input reflection coefficient of the device.

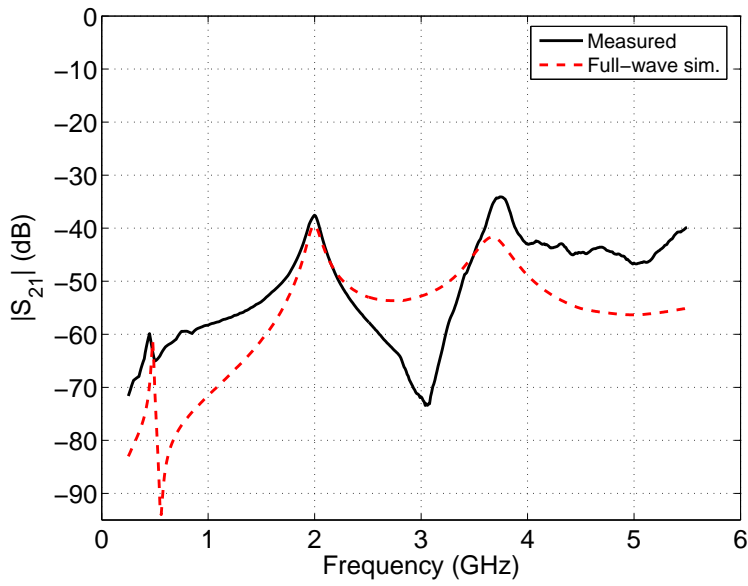

Fig. 9. The magnitude of the simulated and measured transmission coefficient of the device.

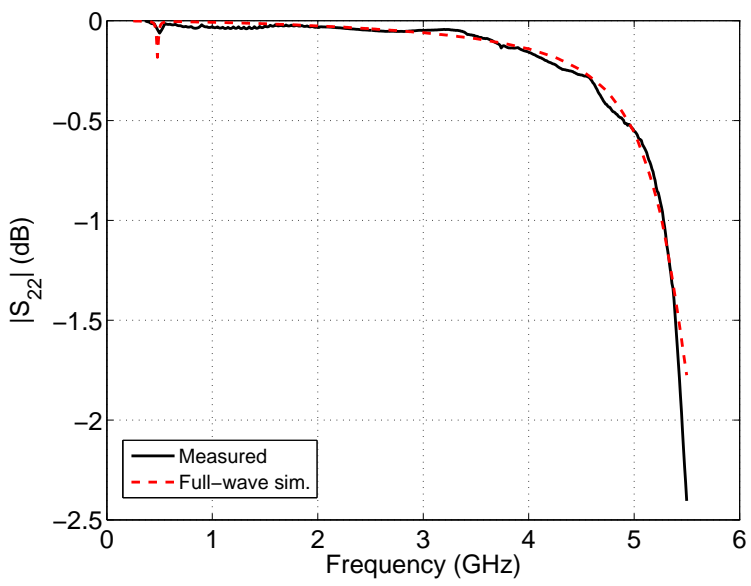

Fig. 10. The magnitude of the simulated and measured output reflection coefficient of the device. 


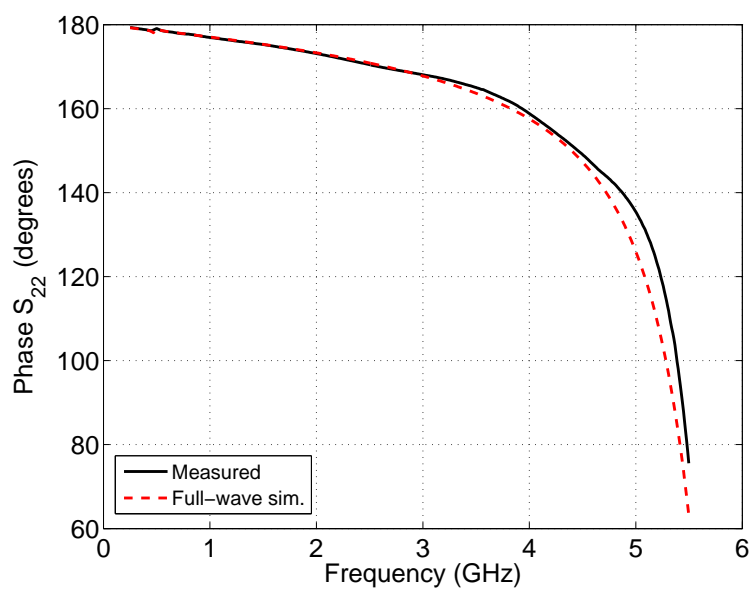

Fig. 11. The phase of the simulated and measured output reflection coefficient of the device.

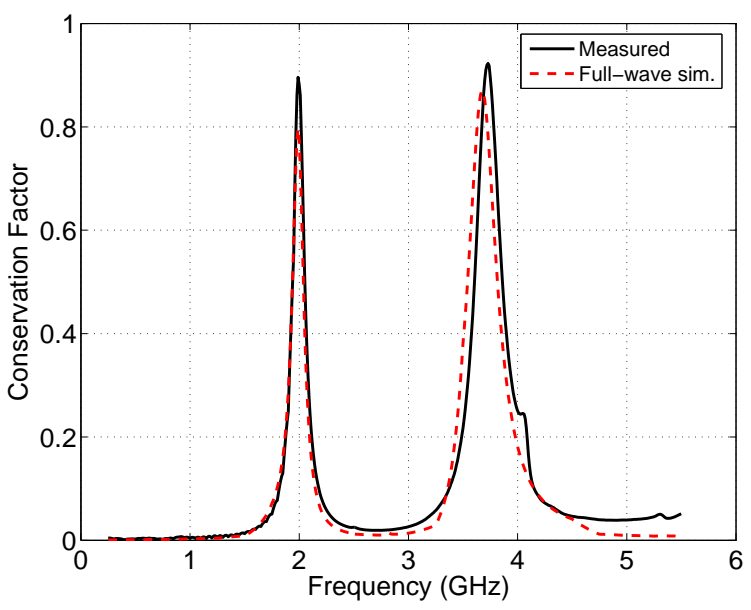

Fig. 12. The measured and simulated conservation factor of the device.

of simulation techniques of the high-power RF/Microwave transistors was also presented. The overall segmentation of the matching network constituent components and the developed simulation methodology were outlined and analyzed. By properly decoupling the sources of inaccuracies of the overall packaged transistor model, an objective assessment on the accuracy of its electrical linear portion a comparison of the measured vs. simulated results was accomplished.

Now that these robust modeling and simulation methodologies and techniques have been used for the examination of the linear portions of the constituent components of the highpower packaged transistor, they can be put into confident use during the design phases of development instead of just being a powerful after-design analysis tools.

\section{ACKNOWLEDGMENT}

The authors would like to express their deepest gratitude to Daren Bridges and Dan Lamey for their thoughtful and insightful discussions, to Eric Shumate for his assistance on measurement related aspects of the work and to John Wood for his assistance with reviewing the content of the manuscript.

\section{REFERENCES}

[1] P. H. Aaen, J. A. Plá, and C. A. Balanis, "On the development of CAD techniques suitable for the design of high-power RF transistors," IEEE Trans. Microwave Theory Tech., vol. 53, no. 10, pp. 3067-3074, Oct. 2005.

[2] D. Brody and G. R. Branner, "A modeling technique for internally matched bipolar microwave transistor networks," in Proc. 37th Midwest Sypm. on Circuits and Systems, Lafayette, LA USA, Aug. 1994, pp. $1224-1226$.

[3] K. Mouthaan, "Modeling of RF high power bipolar transistors," Ph.D. dissertation, Delft University of Technology, Delft, Netherlands, 2001.

[4] T. Johansson and T. Arnborg, "A novel approach to 3-D modeling of packaged RF power transistors," IEEE Trans. Microwave Theory Tech., vol. 47, pp. 760-768, June 1999.

[5] T. Liang, J. A. Plá, P. H. Aaen, and M. Mahalingam, "Equivalentcircuit modeling and verification of metal-ceramic packages for RF and microwave power transistors," IEEE Trans. Microwave Theory Tech., vol. 47, no. 6, pp. 709-712, June 1999.

[6] P. Aaen, J. A. Plá, D. Bridges, and E. Shumate, "A wideband method for the rigorous low-impedance loadpull measurement of high-power transistors suitable for large-signal model validation," in Automatic RF Techniques Group Conference Digest Fall 2000, Boulder, CO, Dec. 2000, pp. 163-169.

[7] K. Goverdhanam et al., "Modeling high power RF LDMOS amplifiers," in 2004 IEEE MTT-S Int. Microwave Symp. Dig., Fort Worth, TX USA, June 2004, pp. 1069-1072.

[8] K. C. Gupta, "Emerging trends in millimeter-wave CAD," IEEE Trans. Microwave Theory Tech., vol. 46, no. 6, pp. 747-755, June 1998.

[9] P. Aaen, J. A. Plá, and C. A. Balanis, "Increased feedback due to package mounting," in 13th Topical Meeting on Electrical Performance of Electronic Packaging, Portland, OR, Dec. 2004, pp. 49-52.

[10] M. E. Goldfarb and A. Platzker, "Losses in GaAs microstrip," IEEE Trans. Microwave Theory Tech., vol. 38, no. 12, pp. 1957-1963, Dec. 1990.

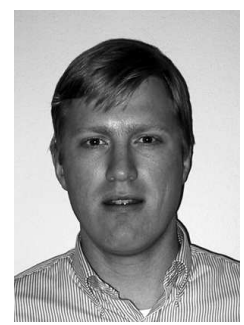

Peter H. Aaen (S93-M97) received the B.A.Sc. degree in engineering science, the M.A.Sc. degree in electrical engineering both from the University of Toronto, Toronto, ON., Canada, and the Ph.D degree in electrical engineering from Arizona State University, Tempe, in 1995, 1997 and 2005, respectively. In 1997, he joined the Wireless Infrastructure Systems Division, Semiconductor Products Sector, Motorola (now Freescale Semiconductor Inc.), in Phoenix, Arizona. His current work focuses on the development and validation of microwave transistor models and passive components. His technical interests include calibration techniques for microwave measurements and the development of package modeling techniques. 


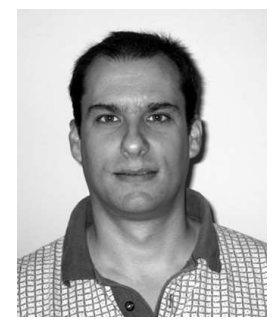

Jaime A. Plá (S'87-M'91) was born in Puerto Rico, in 1969. He received the B.S. degree in electrical engineering from the University of Puerto Rico, Mayagüez, in 1991, and the M.S. degree in microwave engineering from the University of Massachusetts at Amherst, in 1993. In 1991, he joined the Microwave Semiconductor Laboratory, Research Division, Raytheon, Lexington, MA. While with Raytheon, he was primarily involved with the development of microwave measurement techniques and linear and nonlinear models for monolithicmicrowave integrated-circuit (MMIC) semiconductor devices such as GaAs MESFET's PHEMT's, and HBT's. In 1995, he joined the Wireless Infrastructure Systems Division, Semiconductor Product Sector, Motorola (now Freescale Semiconductor Inc.), Phoenix, AZ, where his current work is centered on the development of high-power RF electro-thermal device models for LDMOS devices. Other areas of current interest are the development of package modeling techniques and modeling of passive components, as well as techniques for the measurement of electrical and thermal transistor characteristics related to small- and large-signal modeling extraction and validation.

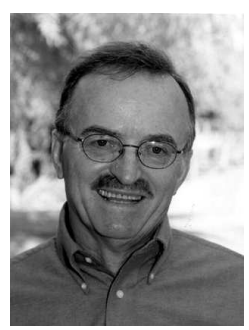

Constantine A. Balanis (S62-M68-M74-F'86LF'04) received the B.S.E.E. degree from Virginia Tech., Blacksburg, the M.E.E. degree from the University of Virginia, Charlottesville, and the Ph.D. degree in electrical engineering from Ohio State University, Columbus, in 1964, 1966, and 1969, respectively. From 1964 to 1970, he was with the National Aeronautics and Space Administration (NASA) Langley Research Center, Hampton, VA. From 1970 to 1983 , he was with the Department of Electrical Engineering,West Virginia University, Morgantown. Since 1983, he has been with the Department of Electrical Engineering, Arizona State University (ASU), Tempe, where he is now Regents' Professor. $\mathrm{He}$ is the author of Antenna Theory: Analysis and Design (New York: Wiley, 2005, 1997 and 1982) and Advanced Engineering Electromagnetics (New York:Wiley, 1989). His research interests are in low- and highfrequency computational methods for antennas and scattering, smart antennas for wireless communication, and high intensity radiated fields (HIRF). Dr. Balanis received the 1987 Graduate Teaching Excellence Award, School of Engineering, ASU, 1989 IEEE Region 6 Individual Achievement Award, 1992 Special Professionalism Award from the IEEE Phoenix Section, 1996 Arizona State University Outstanding Graduate Mentor Award, and 2000 IEEE Third Millennium Medal, an Honorary Doctorate from the Aristotle University of Thessaloniki (AUTH) in 2004, and the 2005 Chen-To Tai Distinguished Educator Award of the IEEE AP Society. He served as Associate Editor of the IEEE TRANSACTIONS ON ANTENNAS AND PROPAGATION from 1974-1977 and IEEE TRANSACTIONS ON GEOSCIENCE AND REMOTE SENSING from 1981-1984. He was Editor of the Newsletter for the IEEE Geoscience and Remote Sensing Society from 1982-1983, Second VicePresident of the IEEE Geoscience and Remote Sensing Society in 1984, Chairman of the Distinguished Lecturer Program from 1988-1991, a Member of of the IEEE Antennas and Propagation Society AdCom from 1993-1995 and 1997-1999, and a Distinguished Lecturer for the IEEE Antennas and Propagation Society from 2003-2005. 